\title{
Engineered Socket Study of Signaling through a 4-Helix Bundle: Evidence for a Yin-Yang Mechanism in the Kinase Control Module of the Aspartate Receptor
}

\author{
Kalin E. Swain, Miguel A. Gonzalez, and Joseph J. Falke* \\ Department of Chemistry and Biochemistry, and the Molecular Biophysics Program, University of \\ Colorado, Boulder, CO 80309-0215
}

\section{Abstract}

The chemoreceptors of E. coli and S. typhimurium form stable oligomers that associate with the coupling protein $\mathrm{CheW}$ and the histidine kinase CheA to form an ultra-sensitive, ultra-stable signaling lattice. Attractant binding to the periplasmic domain of a given receptor dimer triggers a transmembrane conformational change transmitted through the receptor to its cytoplasmic kinase control module - a long 4-helix bundle that binds and regulates CheA kinase. The kinase control module comprises three functional regions: the adaptation region possessing the receptor adaptation sites; a coupling region that transmits signals between other regions; and the protein interaction region possessing contact sites for receptor oligomerization and for $\mathrm{CheA} / \mathrm{CheW}$ binding. Based on the spatial clustering of known signal-locking Cys-substitutions and engineered disulfide bonds, the present study develops the yin-yang hypothesis for signal transmission through the kinase control module. This hypothesis proposes that signals are transmitted through the 4-helix bundle via changes in helix-helix packing, and that the helix packing changes in the adaptation and protein interaction regions are tightly and anti-symmetrically coupled. Specifically, strong helix packing in the adaptation region stabilizes the receptor on-state, while strong helix packing in the protein interaction region stabilizes the off-state.

To test the yin-yang hypothesis, conserved sockets likely to strengthen specific helix-helix contacts via knob-in-hole packing interactions were identified in the adaptation, coupling and protein interaction regions. For 32 sockets, the knob side chain was truncated to Ala to weaken the knob-inhole packing and thereby destabilize the local helix-helix interaction provided by that socket. We term this approach a "knob truncation scan". Of the 32 knob truncations, 28 yielded stable receptors. Functional analysis of the signaling state of these receptors revealed 7 lock-off knob truncations, all located in the adaptation region, that trap the receptor in its "off" signaling state (low kinase activity, high methylation activity). Also revealed were 5 lock-on knob truncations, all located in the protein interaction region, that trap the "on" state (high kinase activity, low methylation activity). These findings provide strong evidence that a yin-yang coupling mechanism generates concerted, antisymmetric helix-helix packing changes within the adaptation and protein interaction regions during receptor on-off switching. Conserved sockets that stabilize local helix-helix interactions play a central role in this mechanism: in the on-state, sockets are formed in the adaptation region and disrupted in the protein interaction region, while the opposite is true in the off-state.

\footnotetext{
*To whom correspondence should be addressed: falke@ colorado.edu, Tel (303) 492-3503, Fax (303) 492-5894.

† Support provided by NIH R01 GM-040731 (to JJF)
} 


\section{Keywords}

Bacterial Chemotaxis; Membrane Protein; Chemoreceptor; CheA; Knob-in-Hole Packing

The homodimeric chemoreceptors of bacterial chemotaxis span the inner cell membrane. These receptors bind attractants in the periplasmic space and generate a transmembrane

conformational change that regulates the activities of the cytoplasmic CheA kinase, ultimately controlling the swimming behavior of the bacterial cell and driving cellular chemotaxis up an attractant concentration gradient (reviewed in (1-6)). The oligomeric structure of the chemoreceptors is complex: each receptor homodimer associates with two other dimers to form a receptor trimer-of-dimers $(1,7,8)$ (Figure 1). In turn, these trimers-of-dimers associate to form a hexagonal, 2-dimensional array of trimers (9-11). All of the cytoplasmic components of the chemotaxis pathway assemble onto the receptor array, thereby generating a multi-protein signaling complex $(3,12,13)$. The positive cooperativity between receptors provided by array organization is believed to be crucial to the ultrasensitivity of the pathway, which can detect exquisitely small changes in attractant concentration $(14,15)$. The array organization is also likely to be essential for the recently discovered ultrastability of the isolated signaling complex, which exhibits a structural and functional half life exceeding several days (16)

The chemoreceptor homodimer exhibits a modular structure built from collections of 4-helix bundles $(1,8,17,18)$ (Fig. 1). The periplasmic ligand binding domain is a dimer of two antiparallel 4-helix bundles. Two helices from each subunit associate to form a membranespanning, antiparallel 4-helix bundle, in which the transmembrane signal is generated by the piston-type displacement of the signaling helix $(1,6,19)$. In the cytoplasm, the signaling helix is coupled to the conserved HAMP domain, a parallel 4-helix bundle that transduces the transmembrane piston signal into a different type of conformational change $(1,20,21)$. This unidentified HAMP conformational change in turn regulates the on-off switching of the long, extended 4-helix bundle termed the kinase control module (1).

The present study focuses on the conformational transitions that underlie on-off switching within the kinase control module and its three distinct functional regions: the adaptation region, the coupling region, also termed the flexible region (22), and protein interaction region (1). The adaptation region possesses the conserved Glu residues that are methyl esterified and demethylated by the adaptation enzymes CheR and CheB, respectively, as well as a high density of other anionic residues that generate electrostatic repulsion between and within the helices of the 4-helix bundle (23). The coupling region is less conserved than the other regions (22) except for a conserved Gly hinge that has been shown to enable the bundle to bend (2426). The protein interaction region possesses the highly conserved interaction sites essential for trimer-of-dimer formation, and for binding the coupling protein $\mathrm{CheW}$ and the histidine kinase CheA in the ultrasensitive, ultrastable, signaling complex $(1,25,27-31)$. During signal transduction, conformational signals arising from both attractant binding and covalent modification of the adaptation sites are transmitted through the adaptation, coupling, and protein interaction regions to the bound CheA kinase. The resulting kinase on-off switching controls both the motor regulation and adaptation branches of the pathway, which are each essential for chemotaxis.

Previous Cys and disulfide scanning studies of the adaptation and protein interaction regions have revealed a striking spatial pattern of effects on receptor function (32-37). In the adaptation region one finds the highest density of lock-on disulfide bonds that covalently stabilize helixhelix packing between the two subunits of the homodimer and trap the receptor in its kinaseactivating state $(33,36)$. By contrast, in the protein interaction region one finds the highest density of lock-on Cys substitutions that, in their reduced state, trap the receptor in its kinase- 
activating state, perhaps by weakening helix-helix interactions $(35,37)$. Here we first confirm these earlier conclusions using the recently developed, anti-symmetric activity method that enables positive identification of lock-on and lock-off modifications in the full-length, membrane-bound receptor (19). Based on the observed clustering of lock-on and lock-off modifications in different regions, we propose the yin-yang model for signal transmission between the adaptation and protein interaction regions. The model hypothesizes that the receptor "on" state possesses stable helix-helix packing in the adaptation region, which destabilizes helix-helix packing in the protein interaction region and activates CheA kinase. The reverse configuration is proposed to generate the receptor "off" state that inhibits CheA kinase.

To test the yin-yang hypothesis, a new "knob truncation scan" approach is developed to systematically perturb helix-helix interactions at specific locations by weakening local socket motifs, each of which consists of a knob side chain that inserts into a hole formed by four residues on an adjacent helix $(38,39)$. The approach is applied to conserved sockets in the adaptation, coupling, and protein interaction regions of the full-length, membrane-bound receptor. The observed functional effects of the knob truncations strongly support the yinyang hypothesis in which the helix-helix packing states of the adaptation and protein interaction regions are tightly coupled to each other but exhibit opposite signaling polarities.

\section{Materials and Methods}

\section{Materials}

Reagents were obtained from the following sources: $\left[\gamma^{32} \mathrm{P}\right]$-ATP from Perkin-Elmer; QuickChange site-directed mutagenesis kit from Stratagene; mutagenic oligonucleotides from Integrated DNA Technologies; all other analytical grade chemicals from Sigma unless noted otherwise. E. coli strains were graciously provided by Dr. J. S. Parkinson (University of Utah).

\section{Identification of Conserved Sockets Likely to Contribute to Helix-Helix Packing Stability}

To select conserved sockets likely to stabilize helix-helix packing in the kinase control module, the program SOCKET by Derek Woolfson and coworkers (39) was used to predict stable knobin-holes packing interactions both in (i) the well-defined coupling and protein interaction regions of the published crystal structure ((8,17), PDB 1QU7, residues 323-462 and 323'-462' of Tsr corresponding to residues $321-460$ and $321^{\prime}-460^{\prime}$ of Tar, respectively), and (ii) the modeled structure of the adaptation region based on chemical constraints $(8,17,33,34)$, residues $262-322,262^{\prime}-322^{\prime}, 463-522$ and $463^{\prime}-522^{\prime}$ of Tsr corresponding to residues 260-320, 260'$320^{\prime}, 461-520$ and $461^{\prime}-520^{\prime}$ of Tar, respectively). A cutoff value of $7.8 \AA$ was used to include both strong and relatively weak sockets. Subsequently, an alignment of related receptor sequences (22) was used to determine which sockets were most conserved, and thus most likely to contribute significantly to helix-helix packing stability. Finally, sockets in which the knob was Ala were eliminated, yielding a library of conserved sockets with knobs all larger than Ala.

\section{Creation and Isolation of Mutant Aspartate Receptors}

Site-directed mutagenesis was performed as previously described (28) using the PCR-based QuickChange mutagenesis kit to engineer point mutations into the Salmonella typhimurium aspartate receptor gene in plasmid pSF6 (40). Mutated plasmids were transformed into $E$. coli strain RP3808 lacking the major endogenous receptors and other pathway components, expressed, then receptor-containing membranes were isolated, quantitated and stored as previously described (23) except that EDTA was reduced from $10 \mathrm{mM}$ to $0.1 \mathrm{mM}$ in the high salt buffer. The final membranes were resuspended at a receptor concentration of $\sim 50 \mu \mathrm{M}$ in 
EDTA-free final buffer ( $20 \mathrm{mM}$ sodium phosphate, $\mathrm{pH} 7.0$ with $\mathrm{NaOH}, 10 \%$ glycerol, and 0.5 $\mathrm{mM}$ phenylmethylsulfonylfluoride).

\section{Oxidation of Receptors to Form Disulfide Bonds}

Disulfide formation reactions were carried out for receptor-containing membranes $(6 \mu \mathrm{M}$ receptor dimer) in Buffer A (50 mM Tris pH 7.2 with $\mathrm{HCl}, 5 \mathrm{mM} \mathrm{MgCl}_{2}$, and $160 \mathrm{mM} \mathrm{KCl}$ ). Reactions were initiated by adding the redox catalyst $\mathrm{Cu}(\mathrm{II})(1,10 \text {-phenanthroline })_{3}(0.2$ or 2 $\mathrm{mM}$ as indicated) in the presence of ambient-dissolved oxygen $(200 \mu \mathrm{M})$. No EDTA was used to buffer the catalyst. Parallel reactions containing the reducing agent DTT $(75 \mathrm{mM})$ instead of redox catalyst were carried out to enable comparisons between the fully disulfide-linked and fully reduced states. All reactions were incubated for $20 \mathrm{~min}$ at $37^{\circ} \mathrm{C}$, then were placed on ice and immediately divided and reconstituted with CheA and CheW (both Cysless) for kinase assays, or with CheR for methylation assays (assays described below). Part of each sample was quenched by mixing with $4 \mathrm{X}$ Laemmli non-reducing sample buffer containing 10 mM EDTA and $40 \mathrm{mM} \mathrm{N}$-ethyl maleimide (NEM) and was immediately heated to $95^{\circ} \mathrm{C}$ for 1 min to promote unfolding; then products were resolved by Laemmli SDS-PAGE and quantitated by densitometry of the Coomassie-stained protein bands as previously described $(20,23)$. The oxidation conditions used herein were considerably stronger than those used in early studies (33-37), enabling disulfide formation to be driven to completion. These stronger oxidation conditions were made possible due to the use of Cysless CheA and CheW proteins (28): in the past, such oxidation conditions were not possible because they interfered with kinase assays employing wild type $\mathrm{Che} \mathrm{A}$ and $\mathrm{CheW}$, both of which contain native Cys residues. CheR also contains native Cys residues but is less sensitive to oxidation: membranes exposed to the strongest oxidation conditions ( $2 \mathrm{mM}$ catalyst) inhibited maximum CheR activity approximately $25 \%$. To correct for this effect, CheR activities were normalized to that measured for WT receptor membranes treated with the same oxidation conditions.

\section{Receptor Activity Assays}

Mutant receptors were assessed for their ability to regulate chemotaxis in vivo by expressing each receptor in E. coli strain RP8611, a strain lacking the aspartate receptor, then measuring cellular chemotaxis at $30^{\circ} \mathrm{C}$ in a standard soft agar swim plate assay, using minimal media containing $100 \mu \mathrm{M}$ aspartate $(23,41)$. Mutant receptors were assessed for their ability to activate and regulate CheA kinase by reconstituting receptor-containing, isolated membranes with purified CheA and CheW (Cysless constructs as previously described (28)), then quantitating receptor-regulated kinase activity in the standard in vitro assay under conditions where the kinase activity of CheA is the rate-determining step in CheY phosphorylation (28). Mutant receptors were assessed for the ability of their adaptation sites to serve as substrates for methyl esterification by reconstituting receptor-containing membranes with purified Che R in the standard in vitro receptor methylation assay (28). Finally, mutant receptors which failed to significantly activate CheA kinase were tested for CheA binding using a previously described method (16).

\section{Standard Deviation}

Error ranges represent the standard deviation of the mean for $\mathrm{n} \geq 3$.

\section{Results}

\section{Development of the yin-yang model for cytoplasmic domain signaling}

Our study began by reexamining the engineered Cys residues and disulfide bonds previously proposed (33-37) to lock the cytoplasmic kinase control module of the full-length, membranebound aspartate receptor in its native on-state that activates CheA kinase. Two technical 
advances achieved in recent years now make it possible to better characterize the effects of such modifications on receptor signaling. (i) The antisymmetric activity method more rigorously identifies modifications that stabilize the native receptor on- or off-signaling state (19). This method subjects modified receptors to both the receptor-coupled CheA kinase activity assay and the receptor-coupled CheR methylation activity assay. The receptor on-state generates a positive signal in the kinase assay and a negative signal in the methylation assay, while the off-state yields the opposite results. These anti-symmetric activity patterns yield unambiguous identification of the native signaling states. (ii) The availability of Cysless CheA and $\mathrm{CheW}$ proteins (28) now enables the use of stronger oxidation conditions to drive engineered disulfide bonds towards completion, thereby allowing more rigorous analysis of their effects on receptor-regulated kinase activity. Oxidation of native proteins (aspartate receptor, $\mathrm{CheA}, \mathrm{CheW}$ and $\mathrm{CheY}$ ) prior to the kinase assay leads to inhibition of kinase activity (Swain and Falke, data not shown), presumably due to oxidative damage to native Cys residues in Che A and/or CheW since receptor and $\mathrm{CheY}$ both lack Cys residues. By contrast, even strong oxidation conditions yield little inhibition of kinase assays employing Cysless Che A and CheW (Swain and Falke, data not shown). Turning to the methylation assay, the native CheR employed herein contains native Cys residues, but inhibition of this enzyme by the strong oxidation chemistry is minor and easily accounted for by appropriate controls (see Methods). Overall, then, most engineered pairs of receptor Cys residues can be oxidized virtually completely to disulfide prior to functional analysis in both the kinase and methylation assays.

We have employed these two technical advances to carefully reexamine the relevant Cys and disulfide modifications in the kinase control module. As positive controls, a total of 6 signalretaining, lock-on, and lock-off disulfides previously identified in the periplasmic and transmembrane regions $(40,42)$ were tested. These control disulfides each form efficiently under the relatively mild oxidation conditions employed previously and their functional effects have been analyzed by multiple activity assays $(40,42)$, ensuring their suitability as positive controls in the present study. The new findings for the positive control disulfides, summarized in Supplemental Table S1, indicate that the new methods correctly identify the different functional effects of receptor modifications. Application of these methods to modifications located in the kinase control module confirms that 16 previously identified lock-on modifications, namely 11 Cys substitutions and 5 disulfide bonds, do indeed trap the receptor in the on-state as operationally defined both by (i) high, aspartate-insensitive kinase activity and (ii) low, aspartate-insensitive receptor methylation rates (see Supplemental Table S1 and Fig. 4A below). In addition, the new findings reveal a previously unknown lock-on disulfide in the adaptation region (Supplementary Table S1). Overall, a striking spatial segregation of lock-on modifications is observed: 9 of the 11 lock-on Cys residues are located in the protein interaction region, while 5 of the 6 lock-on disulfide bonds are located in the adaptation region (Fig. 4A below).

The observation that lock-on Cys residues are highly concentrated in the protein interaction region, while lock-on disulfides are located primarily in the adaptation region, has led us to propose the yin-yang hypothesis. In this model, signals are transmitted through the 4-helix bundle of the kinase control module via anti-symmetric changes in helix-helix packing that occur in a concerted fashion in the adaptation and protein interaction regions. In the adaptation region, stronger helix interactions within the 4-helix bundle are proposed to stabilize the receptor on-state, and weaker interactions are proposed to stabilize the off-state. This model explains the observation that multiple lock-on disulfides can be generated in the adaptation region, each of which covalently stabilizes helix-helix contacts and constitutively favors the on-state. In addition, the model is consistent with observation that electrostatic repulsion between multiple negative charges in the adaptation region destabilizes helix-helix packing and favors the off-state, while covalent modification (methylation or amidation) of the adaptation Glu residues reduces electrostatic repulsion, stabilizes helix-helix packing, and 
favors the on-state (23). In the protein interaction region, the yin-yang model proposes that helix-helix interactions exhibit the opposite functional effects, such that stronger helix-helix contacts within the protein interaction bundle stabilize the receptor off-state, and weaker contacts stabilize the on-state. This feature explains the observation that multiple Cys substitutions located at helix-helix interfaces in the protein interaction region, which presumably weaken local packing interactions, lock the receptor in the on-state. The yinyang hypothesis is testable, as illustrated by studies described below.

While the present reanalysis has confirmed the previous functional classifications of 22 control and experimental receptor modifications, it has also revealed that several modifications were incorrectly classified in previous studies. Notably, the four adaptation region disulfide bonds previously assigned as signal retaining, and thus believed to retain normal receptor on-off switching (G271C-G271C', S272C-S272C', A304C-A304C', G467C-G467C') (33,36), are not confirmed (Supplemental Table S1). The weaker oxidation conditions employed by previous studies did not drive these disulfides to completion, leading to the misidentification. Here we were able to drive three of the disulfides nearly to completion, yielding altered functional classifications (G271C-G271C' lock-on; S272C-S272C' dead; A304C-A304C' dead; where "dead" is a highly perturbed state characterized by loss of activity in both assays, presumably due to adverse structural effects). The fourth disulfide could not be formed efficiently even with the stronger oxidation conditions (G467C-G467C'). Since there are no longer any known signal-retaining disulfides in the adaptation region, there is no evidence to support the previous suggestion that the conformational change in this region is small enough to be accommodated by the range of disulfide bond conformational flexibility $(33,36)$.

However, given the extensive core hydrophobic interactions throughout most regions of the kinase control module, it is likely that the 4-helix bundle remains largely formed throughout the three regions of the module in both signaling states, which limits the magnitude of helixhelix packing changes during on-off switching. Local defects in 4-helix bundle structure remain possible, and structural studies of thermophillic receptors suggest that local defects may indeed occur (24-26).

\section{Testing the yin-yang model by weakening sockets at helix-helix interfaces: Strategy}

To test the yin-yang model for signaling in the kinase control module, a strategy to weaken local helix-helix interactions and to determine the resulting effects on receptor signaling state was devised. As typical for antiparallel 4-helix bundles, the helix-helix interactions in the kinase control module are stabilized by local "socket" structural elements, in which a "knob" residue on one helix inserts into a cavity formed by four "hole" residues on an adjacent helix $(38,39)$. The component helices exhibit the usual repeating heptad pattern in which the " $a$ " and "d" positions are buried, "b", "c" and "f" are exposed, and "e" and "g" are interfacial. The knob residues are located at "a" and "d" positions, while the hole residues are typically positions "d", "g", "a", "d"; or positions "a", "d", "e", "a", respectively. The strongest sockets possess large, hydrophobic knob and hole residues, although a broad range of socket strengths is created by the diversity of residues found at both knob and hole positions. In general, a selected socket can be weakened by introduction of a small side chain at one or more of its positions, thereby decreasing the surface area of knob-in-hole contacts.

Here we test the yin-yang model by introducing point mutations that replace selected knob side chains with the smaller Ala side chain. For simplicity, these mutations are each termed a "knob truncation". Each knob truncation weakens a socket and thus destabilizes a local helix-helix interaction. Subsequently, the effect of each knob truncation on the receptor signaling state is determined using the anti-symmetric activity assay. The yin-yang model predicts that knob truncations in the adaptation and protein interaction regions will often generate lock-off and lock-on receptors, respectively. An example is illustrated in Figure 2, where a hydrophobic 
knob (L415) in the known structure of the protein interaction region contacts a hydrophobic hole ( $\left(\mathrm{I} 359^{\prime}, \mathrm{I} 362^{\prime}, \mathrm{I}_{363^{\prime}}, \mathrm{I} 366^{\prime}\right)$ in the opposite subunit. Truncation of the Leu to Ala is predicted to significantly reduce the contacts of the knob with its hole residues and weaken the socket. Since the mutation would weaken helix-helix packing in the protein interaction region, the yin-yang model predicts an increased probability the mutant receptor would be locked in its kinase-activating, methylation-inhibiting on-state.

To select a library of suitable knob side chains for truncation to Ala, the program SOCKET by Derek Woolfson and coworkers $(38,39)$ was used to predict the strongest sockets in the published structural model for the kinase control module $(8,17)$ (see Methods). Subsequently, an alignment of related receptor sequences was used to determine which sockets were most conserved, and thus most likely to contribute significantly to helix-helix packing stability (22). Finally, sockets in which the knob was already Ala were eliminated. This procedure yielded the library of 32 conserved aspartate receptor sockets targeted for knob truncation, as summarized in Table 1. The library included 16 sockets in the adaptation region, 8 in the coupling region, and 8 in the protein interaction region. Some knobs insert into a hole on the adjacent helix in the same subunit, while others insert into a hole on an adjacent helix in the other subunit, yielding intra- and inter-subunit sockets, respectively (in Table 1 the hole residues of inter-subunit sockets are indicated by a prime). All but 4 of the 32 knobs are hydrophobic, and the four exceptions are each neutral, hydrogen-bonding side chains (Ser, Asn, Gln) that insert into holes containing at least one other hydrogen bonding side chain. In some cases, a knob residue is also a hole residue for a different conserved socket (Table 1); in principle, truncation of such a residue to Ala will thus weaken two sockets simultaneously and thereby have an even greater destabilizing effect on local helix-helix interactions.

It should be noted that while a high resolution crystal structure of a receptor cytoplasmic domain fragment accurately defines the structures of the coupling and protein interaction regions, the the adaptation region is perturbed in this crystal structure by the truncation of its helices and by non-native crystal packing interactions (PDB 1QU7) $(8,17)$. Thus, the SOCKET analysis utilized the crystal structure coordinates for the coupling and protein interaction regions, but for the adaptation region instead used the coordinates provided by a molecular model of the full length receptor $(8,17)$. The structure of the adaptation region in the latter full receptor model is consistent with chemical studies of adaptation region structure in the full-length, membrane-bound receptor $(8,17,33,34)$. Finally, the fact that each of the 32 of the selected sockets is conserved provides strong support for their correct identification in all three regions of the kinase control module.

\section{Construction of weakened-socket receptor library}

To create a library of mutant aspartate receptors possessing weakened sockets in the kinase control module, PCR site-directed mutagenesis of the aspartate receptor gene tar was used to introduce Ala substitutions at the 32 selected knob positions. The resulting point mutations were confirmed by DNA sequencing. Mutant receptors were expressed in an E. coli strain lacking all major components of the chemotaxis pathway, including the adaptation enzymes (CheB, CheR). The absence of adaptation enzymes ensured that each mutant receptor population possessed identical adaptation sites and thus was homogeneous.

When expressed, 28 of the 32 weakened-socket mutants were stable, membrane-incorporated receptors accounting for $9 \pm 1 \%$ to $14 \pm 1 \%$ of the Total Membrane Protein (TMP). These 28 mutants assemble into trimers-of-dimers since they activate and/or bind Che A at near-normal levels (see below). The remaining 4 mutants located in the adaptation (N317A, V471A, $\mathrm{V} 475 \mathrm{~A}$ ) and protein interaction (V401A) regions exhibited very low yields of membraneassociated receptor $(<1 \%$ TMP) insufficient for further study (Table 1$)$. The strong effects of 
knob truncations at these four positions suggest the corresponding sockets may play an important role in assembly or stability.

\section{Effects of weakened sockets on receptor-regulated CheA kinase activity}

To analyze the effects of the 28 remaining weakened sockets on the receptor signaling state, the behavior of each modified receptor was tested in the anti-symmetric activity assay (19). Of special interest are modifications that lock the receptor in its on- or off-state. In the kinase assay, wherein the receptor is reconstituted with CheA and CheW to generate the functional, membrane-bound signaling complex, lock-on modifications trap the signaling complex in the kinase activating state such that it becomes insensitive to attractant binding. By contrast, lockoff modifications trap the complex in the kinase-inhibiting state.

Table 1 and Figure 3A summarize the effects of weakened sockets on receptor-regulated CheA kinase activity. Strikingly, the effects can be grouped into three distinct classes (Fig. 3A). One class (V268A, Q296A, T303A, V447A, V510A) exhibits wild type-like kinase activity, including nearly complete inhibition by attractant aspartate. A second class (I359A, I363A, V397A, L404A, I415A, I419A, V426A, V450A, M454A) exhibits potential lock-on character because even high, non-physiological levels $(1 \mathrm{mM})$ of aspartate do not fully inhibit kinase activity; instead, the remaining kinase activity is at least 4-fold that of the wild type receptor in its aspartate-occupied state. A third class (V265A, I282A, N286A, L300A, V314A, M349A, I352A, I366A, L376A, I457A, S461 A, I468A, M478A, F513A) exhibits potential lock-off activity, since little or no kinase activation is observed even in the absence of aspartate.

Alternatively, the loss of kinase activation observed for the third class of weakened sockets could arise from failure of the mutant receptor to bind CheA kinase. Thus, the 15 modified receptors that exhibited kinase activities $25 \%$ or less than that measured for wild type receptor were tested for normal complex formation with CheA and $\mathrm{CheW}$ in a pull-down assay (16, 28). The resulting kinase binding data are summarized in Table 1 . All 15 tested receptors bound at least $40 \%$ of the normal level of CheA, indicating that their low kinase activities were not due simply to loss of CheA binding. By contrast, a negative control receptor (N379F) that fails to form trimers-of-dimers (43) exhibited no detectable kinase activity and yielded only $6 \%$ of the normal level of CheA binding, confirming that the assay successfully detects poor CheA binders.

\section{Effects of weakened sockets on receptor-regulated CheR methylation activity}

To positively identify modifications that trap the native receptor on- and off-states, the antisymmetric activity assay also measures the effect on receptor-regulated methylation activity in the reconstituted receptor-CheR complex (19). Lock-on modifications trap the signaling complex in the state exhibiting low methylation activity, such that addition of attractant fails to trigger the native increase in the receptor methylation rate. By contrast, lock-off modifications trap the complex in the state exhibiting high receptor methylation activity, even in the absence of attractant.

Table 1 and Figure 3B summarize the effects of weakened sockets on receptor-regulated CheR methylation activity. Again, the effects can be grouped into three different classes (Fig. 3B). One class (Q296A, M349A, I359A, V447A, M454A) exhibits wild type-like methylation activity yielding a methylation rate within the range $\mathrm{WT} \pm 50 \%$, as well as near-normal attractant regulation in which aspartate triggers a 2- to 4-fold increase in methylation rate. A second class (I282A, N286A, L300A, V314A, S461 A, I468A, M478A) exhibits potential lockoff character, since high levels of methylation are observed even in the absence of aspartate. A third class (V265A, V268A, T303A, I352A, I363A, I366A, L376A, V397A, L404A, I415A, I419A, V426A, V450A, V510A, F513A) exhibits potential lock-on activity, since low levels 
of methylation are observed with little or no activation triggered by a high concentration (1 $\mathrm{mM})$ of aspartate.

\section{Weakened sockets that trap the receptor on- and off-states, and effects on chemotaxis}

Using the standard anti-symmetric activity approach, the combined results of the in vitro kinase and methylation assays identify a set of 12 weakened sockets that trap receptor (44) in one of two states, operationally defined as the on- or off-state, as summarized in Table 1,Figure 3 and Figure 4. Presumably these states correspond to the on- and off-states exhibited by the native, two-state receptor (44). Five weakened sockets, all in the protein interaction region (I363A, V397A, L404A, I415A, I419A) simultaneously yield both high kinase activity and low methylation activity, regardless of whether attractant is present or not, indicating that these sockets trap the receptor in the on-state. Seven weakened sockets, all in the adaptation region (I282A, N286A, L300A, V314A, S261A, I468A, M478A), yield low kinase activity and high methylation activity irrespective of attractant level, indicating these sockets trap the off-state. By contrast, while most weakened sockets in the adaptation and protein interaction regions have large effects on kinase and/or methylation activities, in the coupling region most weakened sockets have only minor effects on both assays and no lock-on or lock-off modifications are observed in this region (Table 1, Figure 3).

Analysis of the effects of weakened sockets on receptor-regulated chemotaxis in live cells, summarized in Table 1, reveals that lock-on and lock-off modifications generally block chemotaxis as expected. In 4 of 5 cases, the lock-on modifications virtually eliminate receptorregulated live cell chemotaxis. Similarly, in 5 of 7 cases, the lock-off modifications block live cell chemotaxis. The simplest explanation for these strong effects on chemotaxis is that the signal-locking character of these 9 weakened sockets is retained in vivo, thereby preventing the receptor on-off switching that is essential for pathway function. The remaining 3 exceptions (L300A, S461A, L404A) exhibit signal-locking character in vitro but display more normal function in vivo, suggesting that in live cells the native $\mathrm{CheB} / \mathrm{CheR}$ adaptation system (absent in the in vitro experiments) can partially repair the signal-locking defects of these 3 weakened sockets. Such repairs are not surprising, since is well established that the adaptation system can correct certain receptor defects and thereby maintain pathway function $(33,35,36,40)$.

\section{DISCUSSION}

Overall, the present study illustrates the usefulness of the knob truncation scan in testing the role of helix-helix interactions in protein function and mechanism. This new method targets the knob side chains of conserved sockets that stabilize helix-helix interactions via knob-inholes packing $(38,39)$. The method introduces a point mutation that shortens, or truncates, a given knob side chain to the smaller Ala, thereby reducing knob-in-hole contacts and weakening the socket. The weakened socket, in turn, weakens the local helix-helix packing interaction. It is difficult to directly determine the effects of a given knob truncation on helix packing stability, and some knob truncations will likely not have the intended effects. Thus, a large library of knob truncations is essential to correctly identify spatial patterns. The functional effects of $28 \mathrm{knob}$ truncations tested herein yield a striking spatial pattern that strongly supports the yin-yang hypothesis as illustrated in Figure 4. In the adaptation region, 7 of 13 knob truncations exhibit lock-off character that traps the receptor in its native kinase-inhibiting state, both in the absence and presence of attractant. In the protein interaction region, 5 of $8 \mathrm{knob}$ truncations exhibit lock-on character that stabilizes the native kinase-activating state, regardless of the attractant concentration. No signal locking mutations are detected in the coupling region. Thus, the opposing signaling polarities predicted by the yin-yang hypothesis for the adaptation and protein interaction regions are directly observed: weaker helix-helix 
packing in the adaptation region favors the receptor off-state, while weaker packing in the protein interaction region favors the on-state.

In both regions, signal locking mutations are observed at intra-subunit sockets and inter-subunit sockets (Table 1). It follows that knob-in-hole interactions between helices in the same subunit, or between helices in different subunits of the same dimer, can both modulate yin-yang coupling and receptor on-off switching. Interestingly, the $28 \mathrm{knob}$ truncations at conserved socket positions examined in detail here all retain significant CheA binding, indicating they all form the trimer-of-dimer oligomers needed to form stable receptor-CheA-CheW complexes $(1,51$, 43,16 ), including the 14 locked-off or damaged mutants that are unable to activate CheA. Thus, in many cases, disruption of a single socket can shift the delicately tuned receptor signaling bias without destroying protein-protein structural interactions that likely involve large numbers of sockets or other contacts.

A group of previously characterized Cys substitutions and engineered disulfide bonds provide further evidence for the yin-yang signaling mechanism. Of the 6 known cytoplasmic, lock-on disulfide bonds, 5 are located in the 120-residue adaptation region where they covalently stabilize helix-helix interactions ((33,34,36), Fig. 4A and Supplementary Table S1), supporting the yin-yang view that stabilization of helix packing in this region leads to kinase activation. Although they were characterized before we implemented our current anti-symmetric activity assay and CheA kinase binding assay, Cys substitutions in the adaptation region also yield effects on kinase regulation consistent with yin-yang predictions $(33,36)$. Cys substitutions at 18 buried positions in the adaptation region largely abolish kinase activity, while only 2 Cys substitutions at buried positions lock the receptor in the kinase-activating state $(33,36)$. Of the 18 Cys substitutions that eliminate kinase activity, 10 are knob or hole residues at heptad positions "a", "d", "e", or "g" in one of the conserved, strong sockets identified by the present study (Table 1). The remaining 8 lock-off Cys substitutions are also located at heptad positions "a", "d", "e", "g" that may participate in sockets not satisfying the present proximity and conservation cutoffs (see Methods). Thus, most or all of the kinase-inhibiting Cys substitutions in the adaptation region weaken conserved sockets, and thereby confirm the yin-yang prediction that weaker helix-helix interactions should favor the receptor off-state. The 2 lockon Cys substitutions in the adaptation region (Fig. 4A) may also be explained by the yinyang model, since both replace buried Gly positions with the more hydrophobic, less helixdestabilizing Cys side chain that may increase the stability of the adaptation 4-helix bundle sufficiently to trap the on-state.

In the 56-residue protein interaction region, a total of 9 lock-on Cys substitutions have been identified which lock the receptor in its native kinase-activating on-state exhibiting high kinase activity and low methylation activity $((34,35,37)$ and Fig. 4A). Of these 9 lock-on Cys substitutions, 7 are located at knob or hole positions in conserved, strong sockets identified by the present study (Table 1). It follows that the majority of these lock-on Cys substitutions weaken helix-helix packing by truncating knob or hole residues, and thereby stabilize the kinase activating state as predicted by the yin-yang hypothesis. In general, it was not possible to find engineered disulfide bonds in the protein interaction region with easily interpreted functional effects. In this region, all but 2 of the disulfide bonds tested in the anti-symmetric activity assay disrupted native receptor structure, as revealed by simultaneously low kinase and methylation rates, or blocked kinase binding to the receptor, or both (Swain and Falke, unpublished). The only 2 exceptions were a signal retaining disulfide and a lock-on disulfide, both located near the hairpin turn at the extreme cytoplasmic tip of the receptor where they may stabilize structural features of the turn rather than helix-helix packing (Supplementary Table S1).

Notably, compared to the conserved sockets of the adaptation and kinase control regions, the conserved sockets of the 80-residue coupling region are not strongly tied to receptor function. 
As predicted by a bioinformatics analysis (22), helix-helix interactions in the coupling region appear to be weaker since even the strongest sockets in this region utilize residues that are predicted by socket theory to yield suboptimal knob-in-hole packing. Of 12 conserved sockets, 4 possess Ala knobs (and were thus excluded from this Ala substitution study) that generally yield weak sockets, while 4 others possess holes dominated by small hydrophobic or polar residues (Ala, Ser, Asn) that also likely yield weak sockets. Of the 8 knob truncations tested in the coupling region, only 3 strongly perturbed receptor function and none were signallocking (Table 1B). This lack of signal-locking perturbations contrasted sharply with the other two regions: in the adaptation region 54\% of the knob truncations were lock-off, and in the protein interaction region $62 \%$ were lock-on. Such findings are consistent with the hypothesis that the coupling region undergoes a low-energy conformational shift during signal transmission between the adaptation and protein interaction regions, and possesses a low density of stable sockets to minimize the energetic barrier to this shift. We propose this region is essential to link the concerted yin-yang helix packing changes in the adaptation and protein interaction regions, and is not highly flexible and dynamic throughout since such extreme flexibility would be inconsistent with a coupling role. Thus, rather than "flexible region", we term this the "coupling region". The region does contain the conserved Gly hinge, where yinyang signals may alter the hinge angle during receptor on-off switching $(24-26,22,45)$. Presumably, this hinge has evolved to enable tight yin-yang coupling.

Figure 5 summarizes our current working model for signal transduction through the receptor cytoplasmic domain, from the HAMP domain through the three regions of the kinase control module. Signals are initiated by two different types of input stimuli that drive receptor on-off switching $(1,6,23,46)$. (i) Conformational signals triggered by attractant binding to the periplasmic ligand-binding domain cause a structural rearrangment of HAMP, which is in turn transmitted to the kinase control module $(1,6)$. Our current working hypothesis proposes that attractant binding causes a scissors-type displacement of the C-terminal HD2 and HD2' helices of the homodimeric HAMP about a pivot point (Swain and Falke, in preparation). As the scissors open, the displacement of the C-terminal ends of HD2 and HD2', which are coupled to helices CD1 and CD1' at the N-terminus of the kinase control module, strains the 4-helix bundle packing in the adaptation region and favors the receptor off-state (23). (ii) Electrostatic signals originating in the adaptation region, where a high density of anionic side chains lining the subunit interface generates electrostatic repulsion between helices, also weakens the packing of the adaptation region 4-helix bundle and favors the receptor off-state $(1,23,46)$. During adaptation to a higher attractant concentration, neutralization of the receptor adaptation Glu side chains via methylation reduces the electrostatic repulsion between helices, thereby stabilizing helix-helix packing in the adaptation region and favoring the on-state. Thus, in the adaptation region, attractant and adaptation signals generate opposing effects on receptor signaling state by altering helix-helix interactions in opposite ways, as required for pathway function.

The yin-yang hypothesis further proposes that the coupling region faithfully transmits each change in helix packing from the adaptation region to the protein interaction region, where it triggers a helix packing change that is concerted but opposite in sign. The simplest interpretation of the knob truncation scan data is that conserved sockets in the adaptation region stabilize the receptor on-state, while conserved sockets in the protein interaction region stabilize the off-state. Thus, on-signals stabilize sockets and their helix-helix interactions in the adaptation region, but disrupt sockets and helix-helix interactions in the protein interaction region, while off-signals have the reverse effects in the two regions. Such yin-yang coupling requires that the sockets in the two regions, as well as all other helix-helix interactions, be well balanced so that receptor signaling bias is maintained at an optimal level. Energetically, such a system employing competing socket interactions in two regions provides a significant advantage when low energy signals (attractant binding, adaptation site modification) must 
trigger switching to a different signaling state, since the energetic cost of disrupting sockets in one region is largely balanced by the formation of sockets in the other region.

In structural and dynamical terms, the transition of the adaptation region between its on- and off-states appears to involve switching of the local 4-helix bundle between a tightly-packed, less mobile, "frozen" on-state and a more loosely-packed, more mobile, "dynamic" off-state. Such a local frozen-dynamic transition is supported by evidence that (i) in the on-state, local helix packing is stabilized by socket formation and by partial neutralization of repulsive charges at adaptation sites, and (ii) in the off-state, local helix packing is destabilized by loss of socket interactions, and often by increased charge repulsion between helices (present data and (23)). Moreover, a signal-induced increase in local helix dynamics has been directly detected by Cys pairs introduced as collision monitors, which yield more rapid disulfide bond formation in the off-state (23). This local frozen-dynamic transition differs from the global frozen-dynamic transition proposed by an early model for cytoplasmic domain on-off switching (47): in the latter model based on studies of cytoplasmic domain fragments, all three regions of the kinase control module simultaneously undergo the same frozen-dynamic transition described herein for the adaptation region. The present evidence, obtained for the full-length, membrane-bound receptor in its functional signaling complex, disfavors the global frozen-dynamic model since opposite changes in helix-helix packing are observed in the adaptation and protein interaction regions during on-off switching. These different findings for the cytoplasmic domain fragment and the full-length receptor could be explained by the loss of native helix packing constraints in the fragment construct. Such relatively unconstrained fragments may also be able to switch more easily into the on-state, which would explain their ability to generate measurable activation of CheA kinase (48).

One of the two signaling states of the adaptation region may possess a recently described bulge in the 4-helix bundle, observed in a crystal structure of a thermophillic adaptation region (24). The bulge forms when a symmetric, conserved pair of Asn residues forms a bidentate hydrogen bond across the hydrophobic subunit interface within the local region containing the adaptation sites. This pair of Asn residues (positions $\mathrm{N} 485$ and $\mathrm{N}^{4} 85^{\prime}$ in the aspartate receptor) is widely conserved in chemotaxis receptors, including the chemoreceptors of $E$. coli and $S$. typhimurium. In the aspartate receptor, we have shown that a disulfide bond bridging the same two positions (N485C-N485C') locks the receptor in the on-state ((36), Supplementary Table $\mathrm{S} 1)$. The lock-on disulfide is structurally similar to the Asn-Asn' sidechain interaction, suggesting that the latter is formed in the on-state and broken in the off-state, but this simple view could be misleading since the disulfide bond would generate closer helix-helix packing than the Asn-Asn' interaction and thus is not a perfect structural match (24).

In the protein interaction region, it is not yet clear whether on-off switching triggers a local conformational change between two relatively stable states, or a local frozen-dynamic transition. When on-state signals disrupt local socket interactions, the region could adopt different stabilizing interactions yielding an alternate stable conformation (two-conformation model), or the local helix packing could become more dynamic (frozen-dynamic model). More work is needed to distinguish these possibilities. Moreover, the central mechanism of receptormediated kinase regulation remains unanswered: how does the switching of the protein interaction region between its two signaling states regulate CheA kinase activity? Here we propose a plausible but speculative hypothesis, based in part on a recent study of substrate domain mobility in free CheA kinase (49). In this hypothesis, the protein interaction region regulates the dramatic mobility of the substrate domain required for rapid collisions with the catalytic domain during autophosphorylation, and with CheY or CheB during phosphotransfer. In the off-state, the substrate domain is proposed to bind in a cleft where it interacts stably with both the protein interaction region and a distinct inhibitory site on the catalytic domain (50), thereby eliminating its mobility and inhibiting kinase activity. The on-state of the protein 
interaction region is proposed to release the substrate domain from this inhibitory site, thereby restoring domain mobility and kinase activity.

The yin-yang model is likely to be relevant to a large superfamily of prokaryotic receptors: these receptors contain conserved adaptation, coupling and protein interaction regions homologous to the aspartate receptor and likely share the same signal transduction mechanism (22). The model makes further testable predictions; for example, it is likely that the signaling effects of socket modifications will be additive, so that multiple socket mutations with partial lock-on (or lock-off) character will generate signaling states that are more fully locked-on (or -off). Such signal-locking socket modifications may well be useful in future biophysical studies of the mechanical basis for the remarkable coupling between the adaptation and protein interaction regions. By contrast, multiple socket mutations with opposite character should cancel each other out; for example, simultaneous incorporation of socket-weakening mutations in the adaptation and protein interaction regions is predicted to have little effect on receptor signaling state. Future studies will continue to investigate the nature of receptor on-off switching in the active, membrane-bound signaling complex.

\section{Supplementary Material}

Refer to Web version on PubMed Central for supplementary material.

\section{Acknowledgments}

The authors gratefully acknowledge Prof. Robert Hodges, University of Colorado Health Sciences Center, for helpful discussions about socket structure and stability, and Dr. Annette Erbse for helpful comments on the manuscript.

\section{Abbreviations}

\section{NEM}

$$
\text { N-ethyl maleimide }
$$

DTT

dithiothreitol

EDTA

$$
\text { ethylenediaminetetraacetic acid }
$$

\section{$\mathrm{Cu}-\mathrm{Phen}_{3}$}

$$
\mathrm{Cu}(\mathrm{II})-[1,10-\text { phenanthroline }]_{3}
$$

\section{References}

1. Hazelbauer GL, Falke JJ, Parkinson JS. Bacterial chemoreceptors: high-performance signaling in networked arrays. Trends Biochem Sci 2008;33:9-19. [PubMed: 18165013]

2. Tindall MJ, Porter SL, Maini PK, Gaglia G, Armitage JP. Overview of mathematical approaches used to model bacterial chemotaxis I: the single cell. Bull Math Biol 2008;70:1525-1569. [PubMed: 18642048]

3. Bardy SL, Maddock JR. Polar explorations Recent insights into the polarity of bacterial proteins. Curr Opin Microbiol 2007;10:617-623. [PubMed: 18006364]

4. Baker MD, Wolanin PM, Stock JB. Signal transduction in bacterial chemotaxis. Bioessays 2006;28:922. [PubMed: 16369945]

5. Bourret RB, Stock AM. Molecular information processing: lessons from bacterial chemotaxis. J Biol Chem 2002;277:9625-9628. [PubMed: 11779877]

6. Falke JJ, Hazelbauer GL. Transmembrane signaling in bacterial chemoreceptors. Trends Biochem Sci 2001;26:257-265. [PubMed: 11295559] 
7. Studdert CA, Parkinson JS. Crosslinking snapshots of bacterial chemoreceptor squads. Proc Natl Acad Sci U S A 2004;101:2117-2122. [PubMed: 14769919]

8. Kim KK, Yokota H, Kim SH. Four-helical-bundle structure of the cytoplasmic domain of a serine chemotaxis receptor. Nature 1999;400:787-792. [PubMed: 10466731]

9. Khursigara CM, Wu X, Subramaniam S. Chemoreceptors in Caulobacter crescentus: trimers of receptor dimers in a partially ordered hexagonally packed array. J Bacteriol. 2008

10. Briegel A, Ding HJ, Li Z, Werner J, Gitai Z, Dias DP, Jensen RB, Jensen GJ. Location and architecture of the Caulobacter crescentus chemoreceptor array. Mol Microbiol 2008;69:30-41. [PubMed: 18363791]

11. Zhang P, Khursigara CM, Hartnell LM, Subramaniam S. Direct visualization of Escherichia coli chemotaxis receptor arrays using cryo-electron microscopy. Proc Natl Acad Sci U S A 2007;104:3777-3781. [PubMed: 17360429]

12. Sourjik V, Berg HC. Localization of components of the chemotaxis machinery of Escherichia coli using fluorescent protein fusions. Mol Microbiol 2000;37:740-751. [PubMed: 10972797]

13. Maddock JR, Shapiro L. Polar location of the chemoreceptor complex in the Escherichia coli cell. Science 1993;259:1717-1723. [PubMed: 8456299]

14. Bray D, Levin MD, Morton-Firth CJ. Receptor clustering as a cellular mechanism to control sensitivity [see comments]. Nature 1998;393:85-88. [PubMed: 9590695]

15. Segall JE, Block SM, Berg HC. Temporal comparisons in bacterial chemotaxis. Proc Natl Acad Sci U S A 1986;83:8987-8991. [PubMed: 3024160]

16. Erbse AH, Falke JJ. The Core Signaling Proteins of Bacterial Chemotaxis Assemble to Form an Ultrastable Complex. Biochemistry. 2009

17. Falke JJ, Kim SH. Structure of a conserved receptor domain that regulates kinase activity: the cytoplasmic domain of bacterial taxis receptors. Curr Opin Struct Biol 2000;10:462-469. [PubMed: 10981636]

18. Milburn MV, Prive GG, Milligan DL, Scott WG, Yeh J, Jancarik J, Koshland DE Jr, Kim SH. Threedimensional structures of the ligand-binding domain of the bacterial aspartate receptor with and without a ligand. Science 1991;254:1342-1347. [PubMed: 1660187]

19. Miller AS, Falke JJ. Side chains at the membrane-water interface modulate the signaling state of a transmembrane receptor. Biochemistry 2004;43:1763-1770. [PubMed: 14967017]

20. Swain KE, Falke JJ. Structure of the Conserved HAMP Domain in an Intact, Membrane-Bound Chemoreceptor: A Disulfide Mapping Study. Biochemistry 2007;46:13684-13695. [PubMed: 17994770]

21. Hulko M, Berndt F, Gruber M, Linder JU, Truffault V, Schultz A, Martin J, Schultz JE, Lupas AN, Coles M. The HAMP domain structure implies helix rotation in transmembrane signaling. Cell 2006;126:929-940. [PubMed: 16959572]

22. Alexander RP, Zhulin IB. Evolutionary genomics reveals conserved structural determinants of signaling and adaptation in microbial chemoreceptors. Proc Natl Acad Sci U S A 2007;104:28852890. [PubMed: 17299051]

23. Starrett DJ, Falke JJ. Adaptation mechanism of the aspartate receptor: electrostatics of the adaptation subdomain play a key role in modulating kinase activity. Biochemistry 2005;44:1550-1560. [PubMed: 15683239]

24. Pollard AM, Bilwes AM, Crane BR. The structure of a soluble chemoreceptor suggests a mechanism for propagating conformational signals. Biochemistry 2009;48:1936-1944. [PubMed: 19149470]

25. Park SY, Borbat PP, Gonzalez-Bonet G, Bhatnagar J, Pollard AM, Freed JH, Bilwes AM, Crane BR. Reconstruction of the chemotaxis receptor-kinase assembly. Nat Struct Mol Biol 2006;13:400-407. [PubMed: 16622408]

26. Coleman MD, Bass RB, Mehan RS, Falke JJ. Conserved glycine residues in the cytoplasmic domain of the aspartate receptor play essential roles in kinase coupling and on-off switching. Biochemistry 2005;44:7687-7695. [PubMed: 15909983]

27. Bass RB, Miller AS, Gloor SL, Falke JJ. The PICM Chemical Scanning Method for Identifying Domain-Domain and Protein-Protein Interfaces: Applications to the Core Signaling Complex of E. coli Chemotaxis. Methods Enzymol 2007;423:1-24. 
28. Miller AS, Kohout SC, Gilman KA, Falke JJ. CheA Kinase of bacterial chemotaxis: chemical mapping of four essential docking sites. Biochemistry 2006;45:8699-8711. [PubMed: 16846213]

29. Mehan RS, White NC, Falke JJ. Mapping out regions on the surface of the aspartate receptor that are essential for kinase activation. Biochemistry 2003;42:2952-2959. [PubMed: 12627961]

30. Ames P, Parkinson JS. Constitutively signaling fragments of Tsr, the Escherichia coli serine chemoreceptor. J Bacteriol 1994;176:6340-6348. [PubMed: 7929006]

31. Liu JD, Parkinson JS. Genetic evidence for interaction between the CheW and Tsr proteins during chemoreceptor signaling by Escherichia coli. J Bacteriol 1991;173:4941-4951. [PubMed: 1860813]

32. Bass RB, Butler SL, Chervitz SA, Gloor SL, Falke JJ. Use of site-directed cysteine and disulfide chemistry to probe protein structure and dynamics: applications to soluble and transmembrane receptors of bacterial chemotaxis. Methods Enzymol 2007;423:25-51. [PubMed: 17609126]

33. Winston SE, Mehan R, Falke JJ. Evidence that the adaptation region of the aspartate receptor is a dynamic four-helix bundle: cysteine and disulfide scanning studies. Biochemistry 2005;44:1265512666. [PubMed: 16171380]

34. Bass RB, Falke JJ. The aspartate receptor cytoplasmic domain: in situ chemical analysis of structure, mechanism and dynamics. Structure 1999;7:829-840. [PubMed: 10425684]

35. Bass RB, Coleman MD, Falke JJ. Signaling domain of the aspartate receptor is a helical hairpin with a localized kinase docking surface: cysteine and disulfide scanning studies. Biochemistry 1999;38:9317-9327. [PubMed: 10413506]

36. Danielson MA, Bass RB, Falke JJ. Cysteine and disulfide scanning reveals a regulatory alpha-helix in the cytoplasmic domain of the aspartate receptor. J Biol Chem 1997;272:32878-32888. [PubMed: 9407066]

37. Bass RB, Falke JJ. Detection of a conserved alpha-helix in the kinase-docking region of the aspartate receptor by cysteine and disulfide scanning. J Biol Chem 1998;273:25006-25014. [PubMed: 9737956]

38. Walshaw J, Woolfson DN. Extended knobs-into-holes packing in classical and complex coiled-coil assemblies. J Struct Biol 2003;144:349-361. [PubMed: 14643203]

39. Walshaw J, Woolfson DN. Socket: a program for identifying and analysing coiled-coil motifs within protein structures. J Mol Biol 2001;307:1427-1450. [PubMed: 11292353]

40. Chervitz SA, Lin CM, Falke JJ. Transmembrane signaling by the aspartate receptor: engineered disulfides reveal static regions of the subunit interface. Biochemistry 1995;34:9722-9733. [PubMed: 7626643]

41. Adler J. Chemoreceptors in bacteria. Science 1969;166:1588-1597. [PubMed: 4902679]

42. Chervitz SA, Falke JJ. Lock on/off disulfides identify the transmembrane signaling helix of the aspartate receptor. J Biol Chem 1995;270:24043-24053. [PubMed: 7592603]

43. Studdert CA, Parkinson JS. Insights into the organization and dynamics of bacterial chemoreceptor clusters through in vivo crosslinking studies. Proc Natl Acad Sci U S A 2005;102:15623-15628. [PubMed: 16230637]

44. Bornhorst JA, Falke JJ. Evidence that both ligand binding and covalent adaptation drive a two-state equilibrium in the aspartate receptor signaling complex. J Gen Physiol 2001;118:693-710. [PubMed: 11723162]

45. Vaknin A, Berg HC. Direct evidence for coupling between bacterial chemoreceptors. J Mol Biol 2008;382:573-577. [PubMed: 18657546]

46. Lai WC, Beel BD, Hazelbauer GL. Adaptational modification and ligand occupancy have opposite effects on positioning of the transmembrane signalling helix of a chemoreceptor. Mol Microbiol 2006;61:1081-1090. [PubMed: 16879656]

47. Kim SH. "Frozen" dynamic dimer model for transmembrane signaling in bacterial chemotaxis receptors. Protein Sci 1994;3:159-165. [PubMed: 8003953]

48. Ames P, Yu YA, Parkinson JS. Methylation segments are not required for chemotactic signalling by cytoplasmic fragments of Tsr, the methyl-accepting serine chemoreceptor of Escherichia coli. Mol Microbiol 1996;19:737-746. [PubMed: 8820644]

49. Gloor SL, Falke JJ. Thermal domain motions of CheA kinase in solution: Disulfide trapping reveals the motional constraints leading to trans-autophosphorylation. Biochemistry 2009;48:3631-3644. [PubMed: 19256549] 
50. Hamel DJ, Zhou H, Starich MR, Byrd RA, Dahlquist FW. Chemical-shift-perturbation mapping of the phosphotransfer and catalytic domain interaction in the histidine autokinase CheA from Thermotoga maritima. Biochemistry 2006;45:9509-9517. [PubMed: 16878985]

51. Boldog T, Grimme S, Li M, Sligar SG, Hazelbauer GL. Nanodiscs separate chemoreceptor oligomeric states and reveal their signaling properties. Proc Natl Acad Sci U S A 2006;103:11509-11514. [PubMed: 16864771] 
Transmembrane

Signaling Module

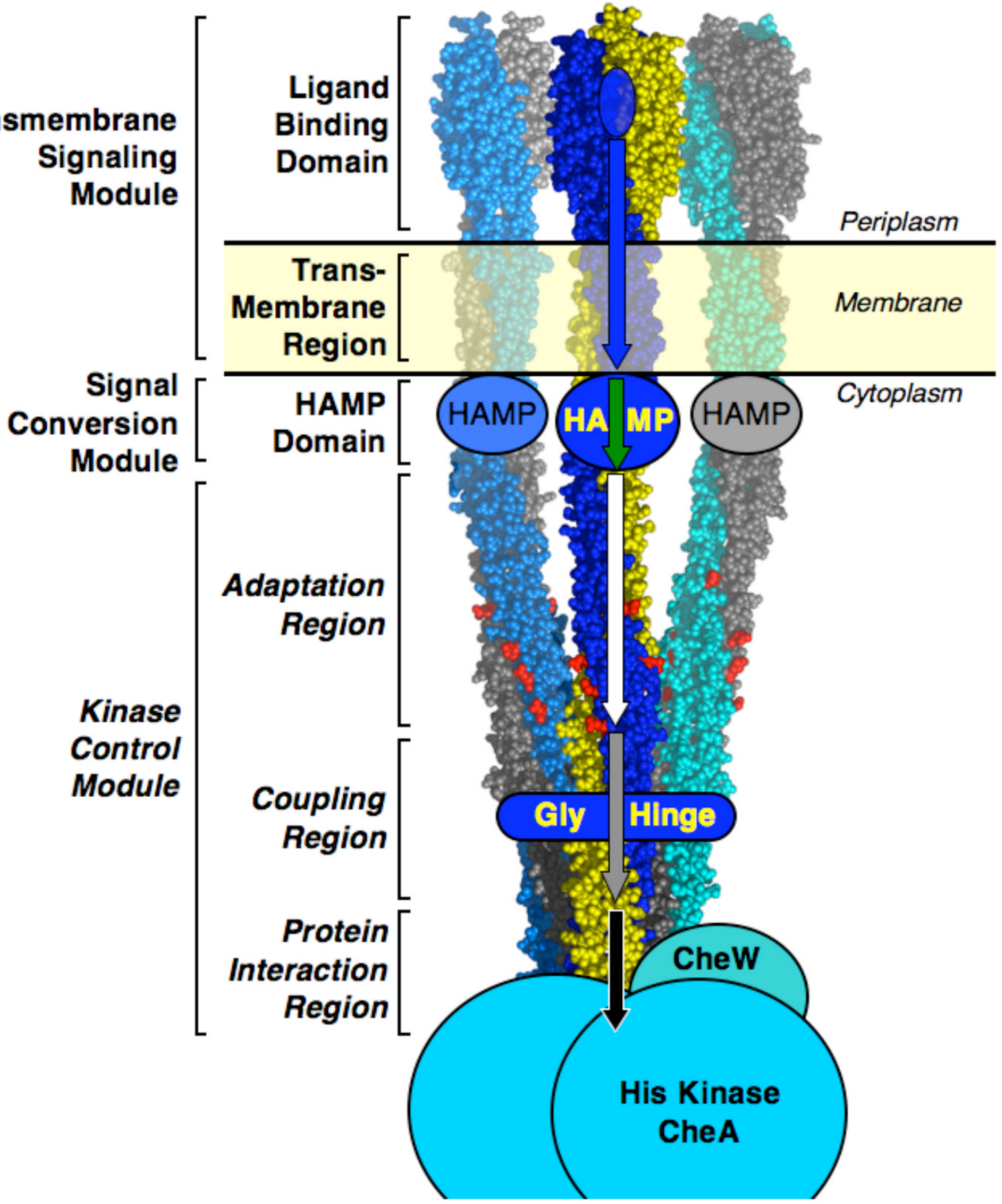

Figure 1. Schematic core complex illustrating different functional regions of the receptor Shown is a receptor trimer-of-dimers (individual subunits are unique colors) in complex with the dimeric histidine kinase CheA (blue) and the coupling protein $\mathrm{CheW}$ (cyan). This core complex is believed to represent the minimal structural unit required for receptor-regulated kinase activity $(1,51)$. The structural-functional regions investigated in the present study are italicized. Attractant binding to the periplasmic ligand binding site is believed to trigger different types of conformational changes in each region denoted by a different arrow color, ultimately transmitting a regulatory signal to the bound CheA kinase. In the transmembrane signaling module, the conformational signal is a piston displacement of the signaling helix (blue arrow). The signal conversion or HAMP module converts this piston displacement into 
a different conformational signal (green arrow). The kinase control module is an extended 4helix bundle that carries information from HAMP to bound CheA and possesses three functional regions. The yin-yang hypothesis developed herein proposes that signal transmission through the regions of the 4-helix bundle involve changes of helix-helix packing, and that the polarities of these packing changes are opposite in the adaptation and protein interaction regions. The coupling region is essential for communication (grey arrow) of these helix packing changes between the adaptation and protein interaction regions. While attractant binding and conformational changes are indicated only in one receptor dimer, positive cooperativity between receptors may generate corresponding conformational changes in the other dimers. 

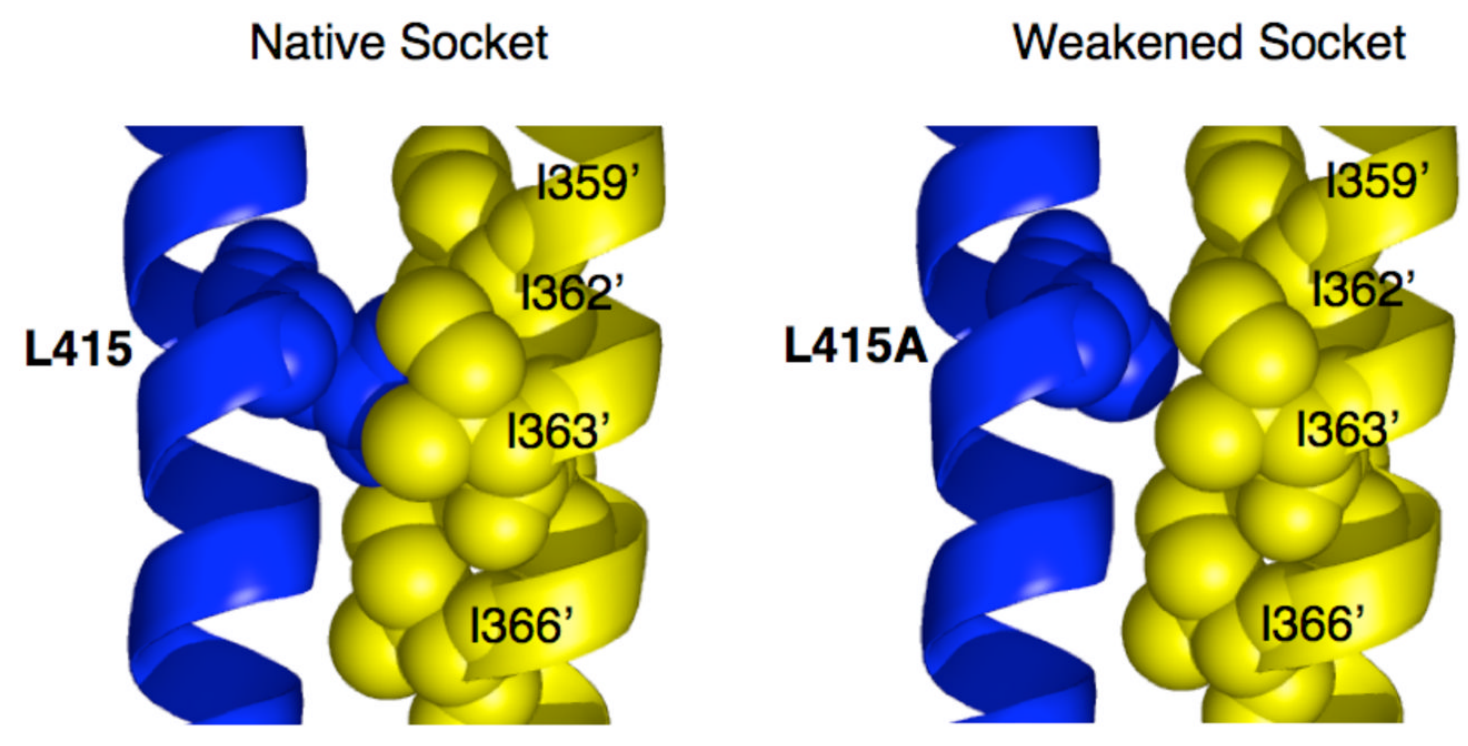

Figure 2. A method to analyze the effects of local helix-helix interactions

Helix-helix packing in 4-helix bundles is stabilized by socket interactions in which a knob side chain inserts into a hole formed by 4 side chains on an adjacent helix $(38,39)$. Thus, to locally reduce the strength of helix-helix packing, in general one can truncate the side chain of a selected knob to Ala, thereby decreasing the knob-in-hole packing of a native, inter-helix socket. Shown is a representative socket (left) in which L415 is the knob that inserts into a hole comprised by I359', I362', I363', and I366' on an adjacent helix provided by the other subunit. Truncating the knob side chain from Leu to Ala, a mutation termed a knob truncation, significantly decreases the knob-hole contacts (right). 


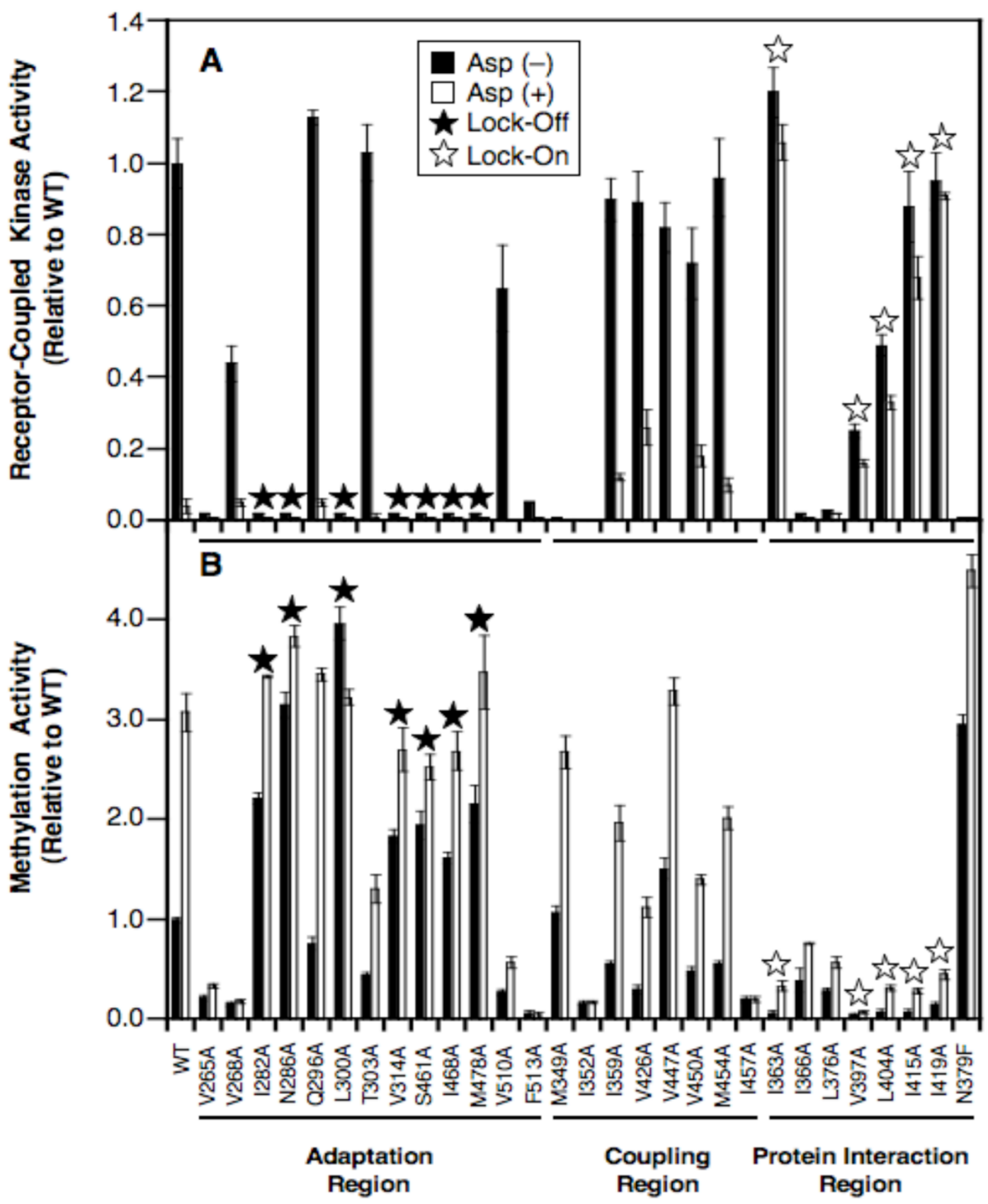

Figure 3. Effects of weakened sockets on receptor function in vitro

A) Effects of knob truncations (defined in Fig. 2) on receptor-regulated kinase activity in the reconstituted receptor-CheA-CheW signaling complex. Shown are CheA kinase activities for signaling complexes containing each knob truncation mutant in both the apo (filled bar) and attractant-occupied (1 mM Asp, open bar) states. All kinase activities are normalized to that of the signaling complex containing the apo wild type receptor. Notably, a high percentage of knob truncations in the adaptation region inhibit kinase activity, while a high percentage in the protein interaction region prevent normal attractant inhibition of kinase activity. B) Effects of knob truncations on receptor methylation rates in the reconstituted receptor-CheR complex. Shown are rates of adaptation site methyl esterification by CheR, both in the apo (filled bar) 
and attractant-occupied (1 mM Asp, open bar) states. All rates are normalized to that of the apo wild type receptor-CheR complex. A high percentage of knob truncations in the adaptation region lead to high methylation rates even in the absence of attractant, while in the protein interaction region the methlation rates are typically low in both the absence and presence of attractant. In addition to the $28 \mathrm{knob}$ truncation mutants, each panel also includes the wild type (WT) receptor and a control receptor $(\mathrm{N} 379 \mathrm{~F})$, which does not form the trimer-of-dimers (43) and thus is inactive in the kinase and live cell assays, but is methylated faster than wild type due to the increased accessibility of the adaptation sites to CheR. Knob truncations that are operationally defined (see text) as signal-locking, either lock-on (open star) or lock-off (filled star), are indicated by stars. 

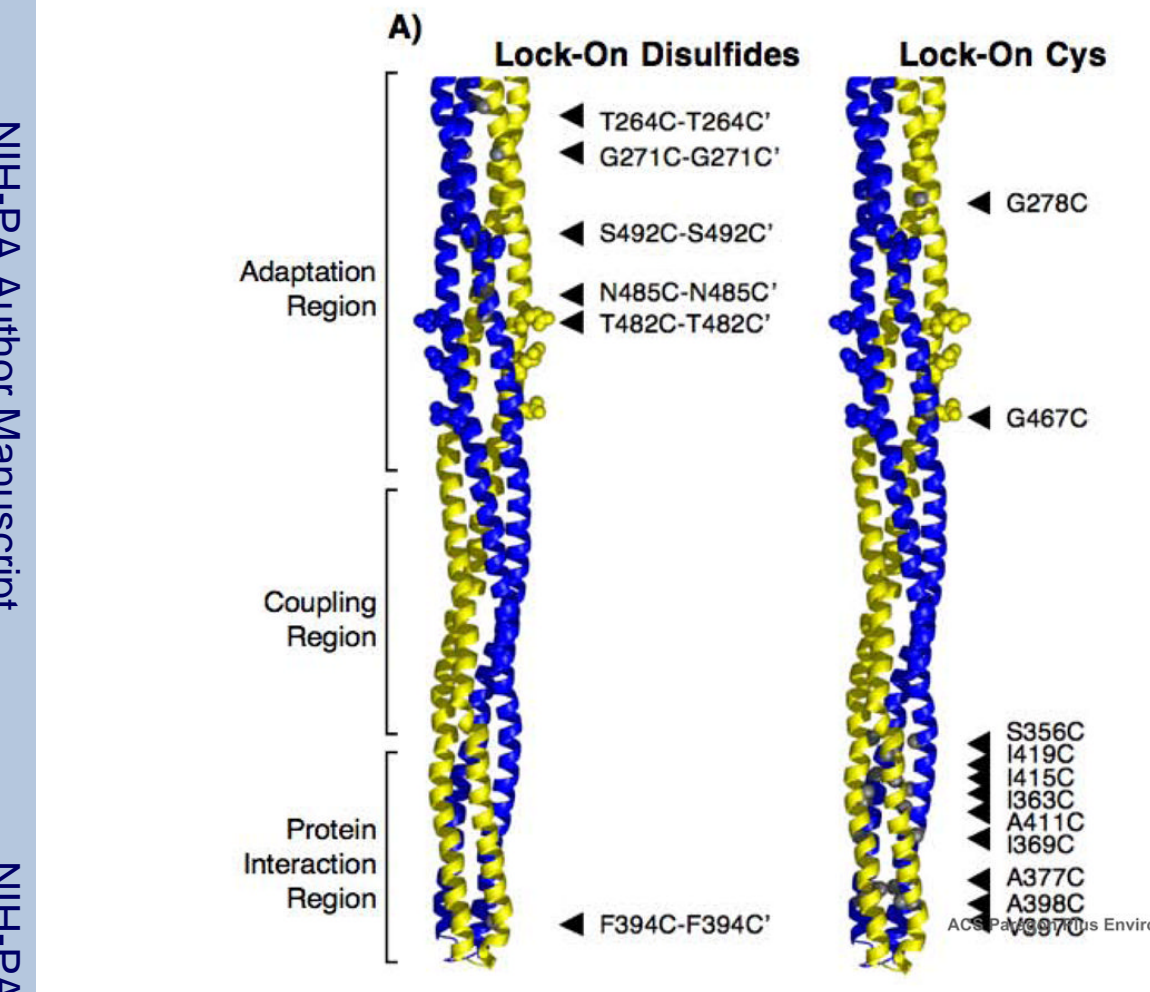

B)

)
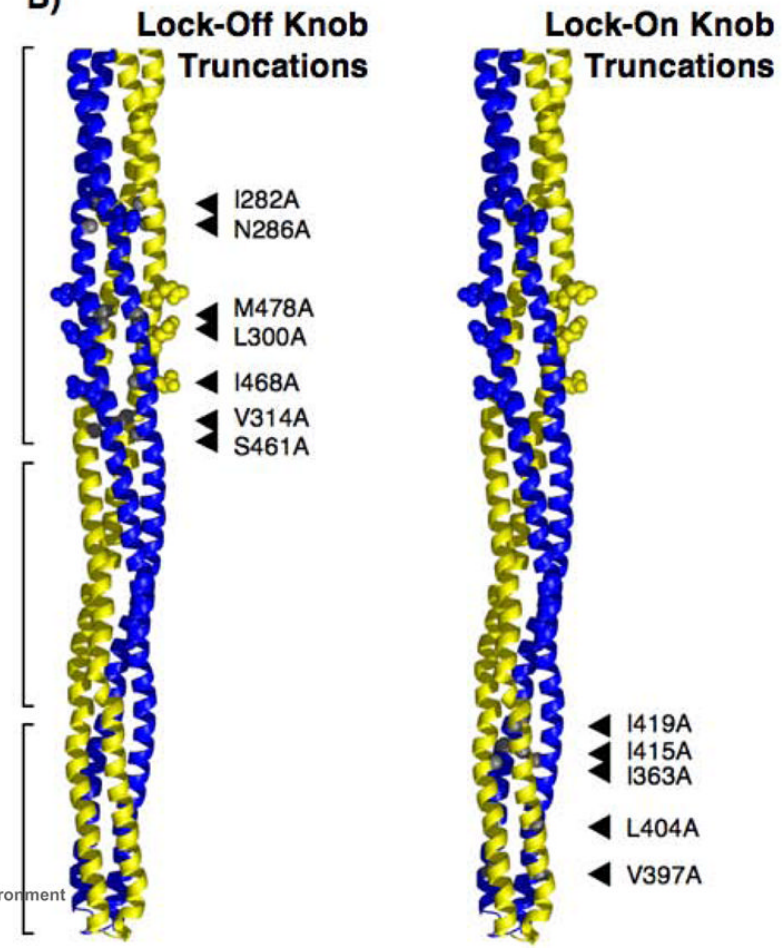

Figure 4. Locations of lock-on and lock-off modifications in the kinase control module Shown is the kinase control module of a single receptor homodimer (1), with the two subunits distinguished by color (blue, gold). Each arrowhead indicates the position of a signal-locking modification (small spheres) in the homodimer, as identified by the anti-symmetric activity assay (see text). Lock-on modifications are operationally defined by high rates of receptorregulated CheA kinase activity, even in the presence of attractant that normally turns the kinase off, as well as low CheR methylation activity regardless of the attractant concentration. Lockoff modifications are defined by low rates of receptor-regulated CheA kinase activity, and high rates of CheR methylation activity, regardless of the attractant concentration. A) The locations of lock-on disulfide bonds and lock-on Cys substitutions, first described in previous studies (33-37) and reconfirmed here using more rigorous methods. The lock-on disulfides covalently stabilize helix-helix packing and are located predominantly in the adaptation region, while the lock-on Cys substitutions likely weaken helix-helix packing and are predominantly located in the protein interaction region. B) The locations of lock-on and lock-off knob truncations, which are clustered in the protein interaction and adaptation regions, respectively. These modifications illustrate the opposite effects that weaker knob-in-holes packing have on receptor signaling state in the two different regions. 
Switching On

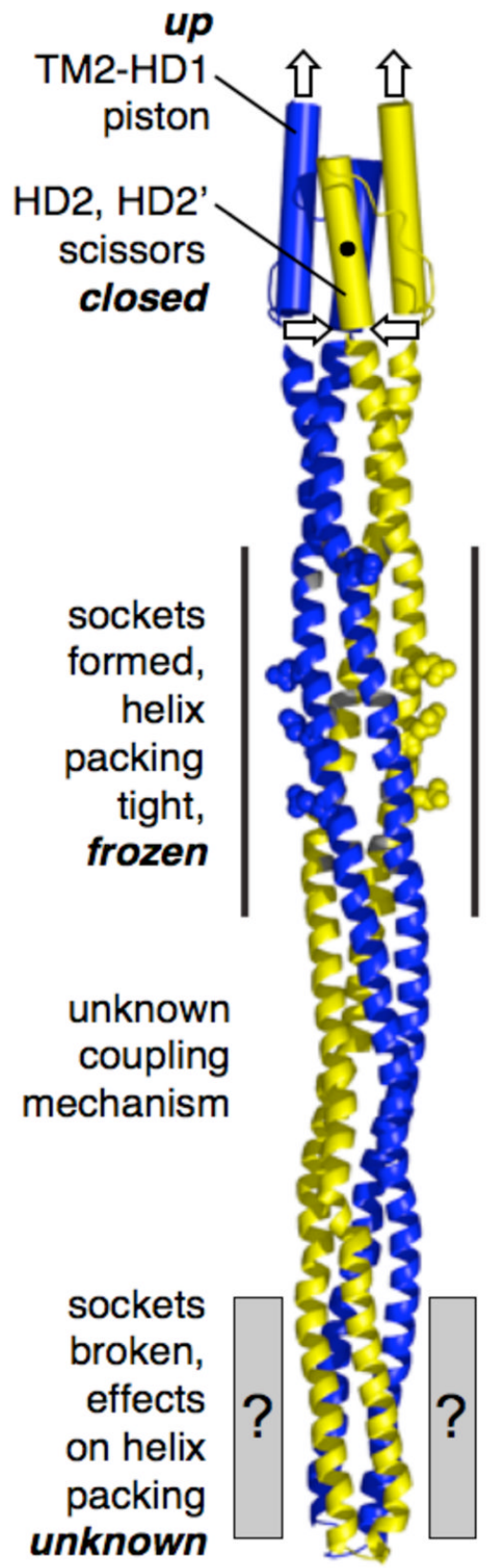

Switching Off

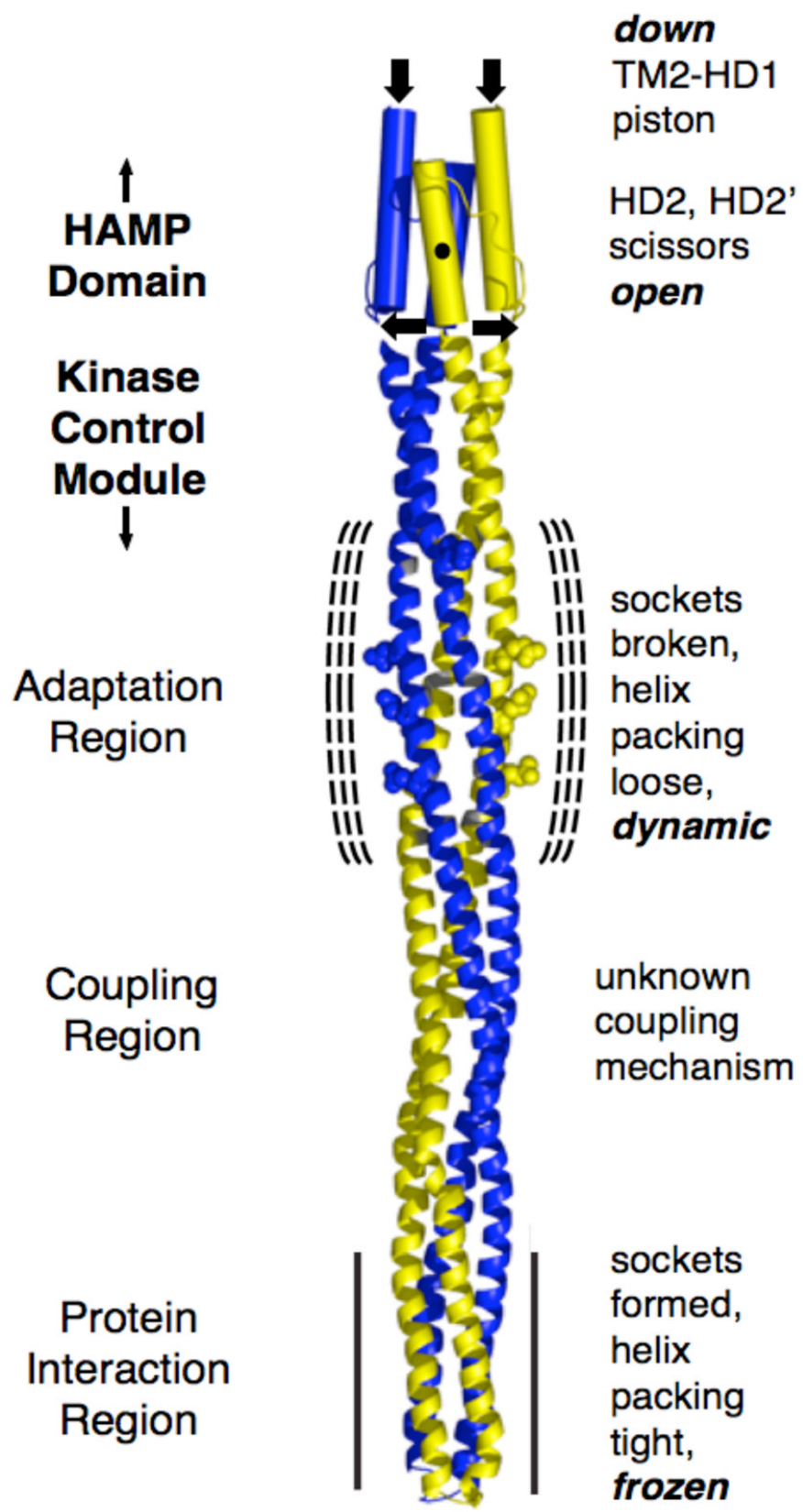

Figure 5. Conceptual basis of the yin-yang hypothesis

While the atomic structural-mechanical basis of the yin-yang signaling is not yet clear, the conceptual basis of the model is straightforward. Attractant binding to the periplasmic ligand binding domain triggers a transmembrane conformational change that is converted by the HAMP domain into a different type of conformational signal (1). Our current working model proposes that HAMP on-off switching triggers a scissors-type displacement of its C-terminal HD2-HD2' helices that directly couple to the N-terminal helices of the kinase control module. In the kinase-activating on-state, 4-helix bundle packing is more stable (sockets formed) in the adaptation region, but less stable (sockets broken, at least partially) in the protein interaction region. The reverse is true in the kinase-inhibiting off-state. Together with previous findings 
on helix dynamics in the adaptation region (23), the present findings indicate that helix packing changes in this region yield a local frozen-dynamic transition (47) as illustrated. In the protein interaction region, no study of helix dynamics has yet been carried out and it is not clear whether the loss of socket interactions (i) allows other stabilizing interactions to form, yielding a different stable conformation, or (ii) simply destabilizes the region, yielding a looser, more dynamic structure. The helix-helix packing in the coupling region is proposed to possess a significantly lower density of stabilizing sockets (22), and the location of this region indicates that it serves as a signal transmission element between the strongly coupled adaptation and protein interaction regions. The space-filling sidechains represent the four adaptation Glu residues of each subunit, which can also drive yin-yang signaling via an electrostatic mechanism (23). 


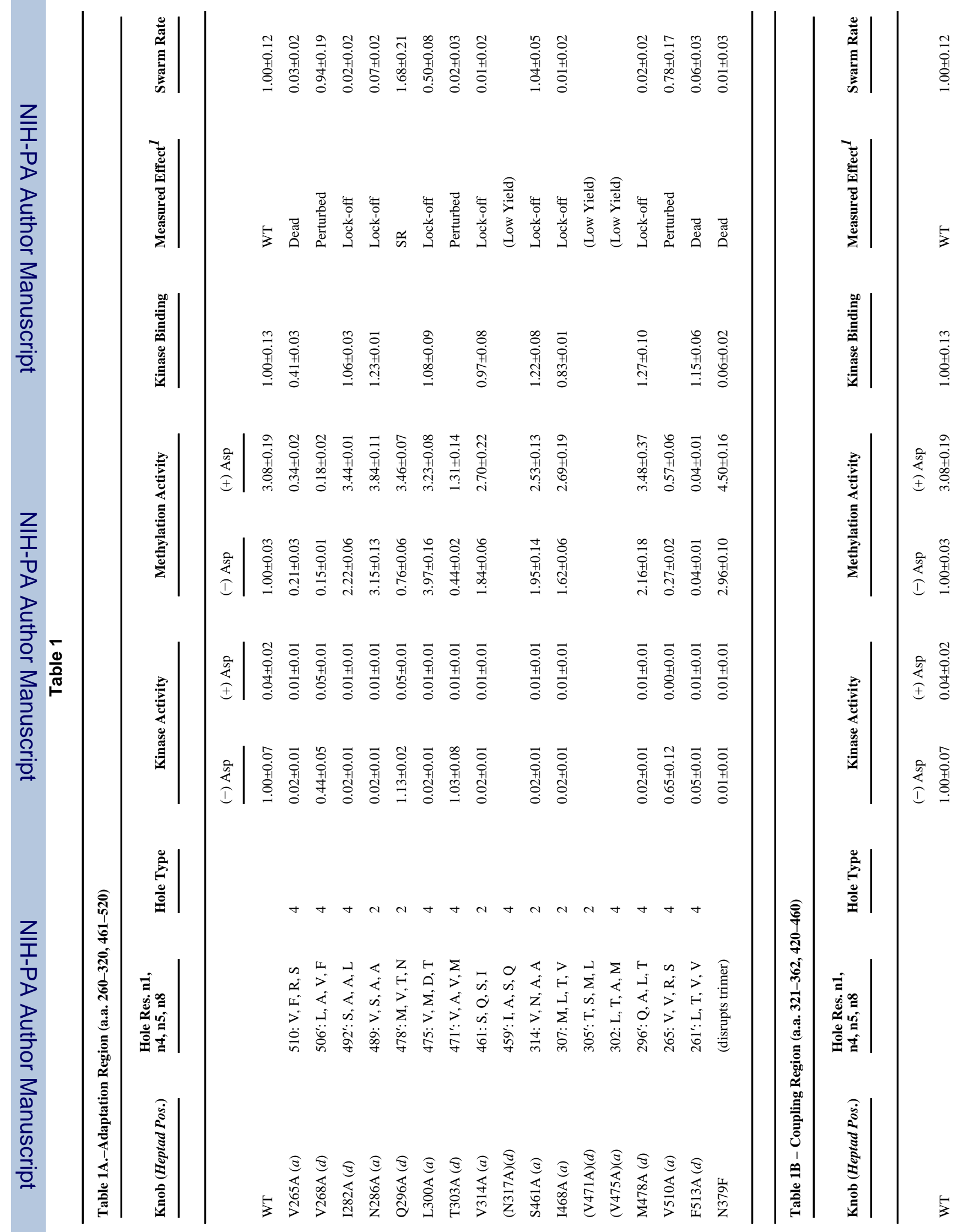

Biochemistry. Author manuscript; available in PMC 2010 October 6. 


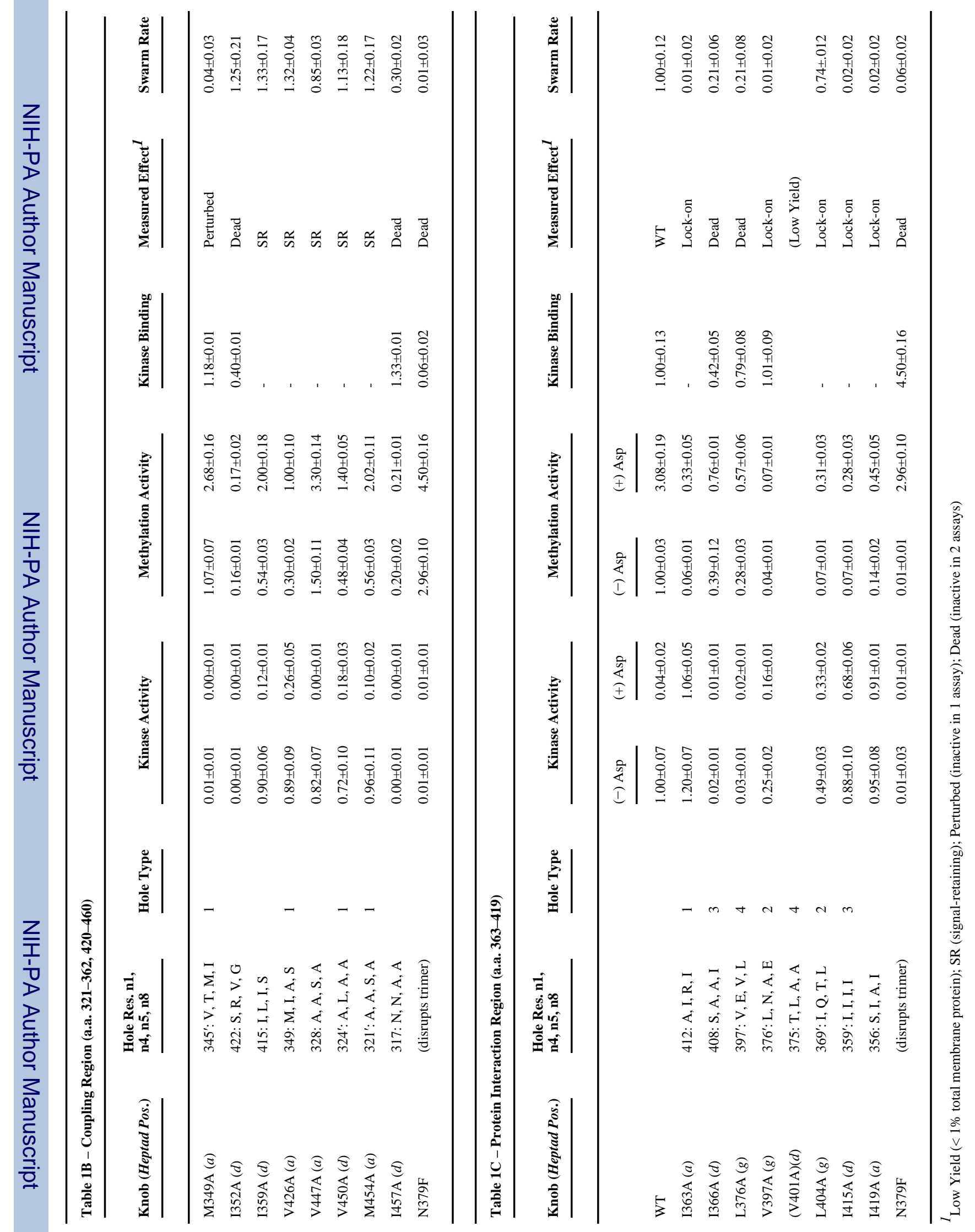

Biochemistry. Author manuscript; available in PMC 2010 October 6. 
